\title{
Die mediale Gonarthrose
}

\section{Arthritis of the Medial Knee Joint Compartment}

G. Matziolis, E. Röhner

\section{Zusammenfassung \\ $\nabla$}

Die Arthrose des Kniegelenks ist mit einer Lebenszeitprävalenz von 23\% bei den über 65-Jährigen eine häufige orthopädische Erkrankung [1]. Aufgrund der demografischen Entwicklung ist mit einer weiteren Zunahme der Anzahl betroffener Patienten zu rechnen. Das mediale Gelenkkompartiment ist dabei aufgrund der Biomechanik und Kinematik des Kniegelenks erheblich häufiger betroffen als das laterale. Erst das Verständnis der funktionellen Anatomie und Pathologie ermöglicht die kritische Wertung der unterschiedlichen zur Verfügung stehenden konservativen und operativen Therapieansätze. Dieser Artikel soll einen Überblick über die diagnostischen und therapeutischen Verfahren der medialen Gonarthrose geben. Dabei sollen insbesondere die medial öffnende tibiale Korrekturosteotomie (HTO), der unikondyläre Gelenkersatz (UNI) und die Totalendoprothese (TEP) als die am häufigsten durchgeführten operativen Maßnahmen vergleichend dargestellt werden. Die aktuelle wissenschaftliche Datenlage wird mit dem Ziel einer evidenzbasierten Diagnostik und einer dem Stadium und der Pathologie angemessenen Therapie der medialen Gonarthrose dargestellt.

\section{Abstract \\ $\nabla$}

$23 \%$ of all persons older than 65 years suffer from osteoarthritis of the medial compartment of the knee joint, a very common situation in orthopaedic practice [1]. As a result of the demographic trend the number of patients is expected to increase in the future. Based on specific joint biomechanics and kinematics the medial knee joint compartment is more frequently affected than the lateral. Only an understanding of the functional anatomy and underlying pathology allows a critical evaluation of different available conservative and operative treatment options. This article gives an overview of diagnostic and therapeutic strategies of osteoarthritis of the medial knee joint. Frequently performed surgeries, e.g. high tibial osteotomy (HTO), unicompartmental knee arthroplasty (UKA) and total knee arthroplasty (TKA) will be presented in a comparative manner. The actual scientific evidence will be given with the goal of an evidence based therapy that is adopted to stage and pathology of osteoarthritis of the medial compartment of the knee joint.
Rubrikherausgeber: R. Hoffmann, Frankfurt R. Windhager, Wien

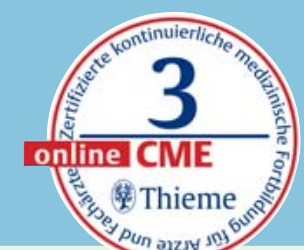

\author{
Schlüsselwörter \\ - Gonarthrose \\ - Korrekturosteotomie (HTO) \\ - unikondyläre Endoprothese (UNI) \\ - Totalendoprothese (TEP) \\ Keywords \\ - knee joint arthritis \\ - corrective osteotomy \\ - high tibial osteotomy (HTO) \\ - unicompartmental knee arthro- \\ plasty (UKA) \\ - total knee arthroplasty (TKA)
}

AWMF Arbeitsgemeinschaft der Wissenschaftlichen Medizinischen Fachgesellschaften

CORA Center of Rotation and Angulation

CPM Continuous passive Motion

HKB hinteres Kreuzband

HTO High tibial Osteotomy (Umstellungsosteotomie)

LIA lokale Infiltrationsanästhesie

NSAID Nonsteroidal anti-inflammatory Drug (nichtsteroidales Antiphlogistikum

OARSI Osteoarthritis Research Society International

PCA Patient controlled Analgesia (patientenkontrollierte Analgetikaapplikation)

TEP Totalendoprothese

UNI unikondyläre Prothese

VKB vorderes Kreuzband

VNR

2760512015147120279

Bibliografie

DOI http://dx.doi.org/ 10.1055/s-0035-1557834 Z Orthop Unfall 2015; 153 : 553 - 566 (c) Georg Thieme Verlag KG Stuttgart · New York . ISSN 1864-6697

Korrespondenzanschrift Univ.-Prof. Dr.med. Georg Matziolis Orthopädische Klinik Friedrich-Schiller-Universität Campus Eisenberg Klosterlausnitzer Straße 81 07607 Eisenberg E-Mail: g.matziolis@ krankenhaus-eisenberg.de 
- Die Bewegung des Kniegelenks ist eine Kombination aus Rollen, Drehen und Gleiten. Dies ist möglich durch die Inkongruenz der Gelenkflächen von Femur und Tibia.

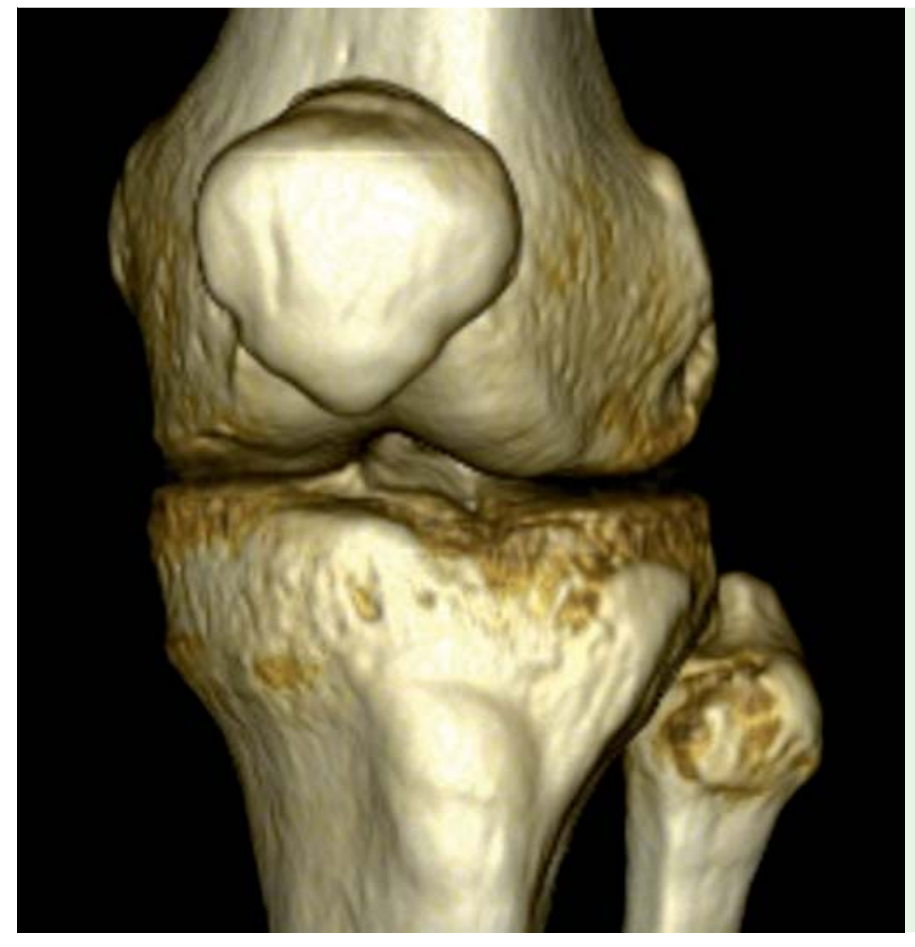

Abb. 1 3-D-CT-Rekonstruktion eines Kniegelenks.

\section{Funktionelle Anatomie und Biomechanik des Kniegelenks \\ $\nabla$}

Die funktionelle Anatomie des Kniegelenks ist für das Verständnis von Genese, Diagnostik und Therapie der medialen Gonarthrose entscheidend. Das Kniegelenk besteht aus Femur, Tibia und Patella ( Abb. 1). Die Fibula ist zwar nicht gelenkbildend, nimmt aber durch den Ansatz des Seitenbands und des M. biceps femoris Einfluss auf die Funktion des Gelenks.

Das Kniegelenk führt stets eine kombinierte Bewegung aus Rollen, Drehen und Gleiten aus. Dies wird erst durch die Inkongruenz der Gelenkflächen von Femur und Tibia möglich.

- Die Femurkondylen sind medial und lateral konvex und besitzen eine unterschiedliche Größe und Geometrie.

- Das mediale Tibiaplateau ist konkav, das laterale konvex.

Die Menisci gleichen diese Inkongruenz der Gelenkpartner anteilig aus. Während der mediale Meniskus am Innenbandkomplex fixiert ist und daher eine geringe Mobilität aufweist, ist der Außenmeniskus beweglich und gleicht die lateral ausgeprägte bikonvexe Inkongruenz aus.

Die Kinematik des Kniegelenks wird durch aktive und passive Stabilisatoren geführt (s. Übersicht).

\section{Übersicht}

Die Stabilisatoren des Kniegelenks

Passive Stabilisatoren:

- Seitenbänder

- Kreuzbänder

- Tractus iliotibialis

- posteriore Gelenkkapsel

Aktive Stabilisatoren:

- M. quadriceps

M. biceps femoris

- M. popliteus

- Mm. gastrocnemii

M. semitendinosus

- M. semimembranosus

- M. gracilis 


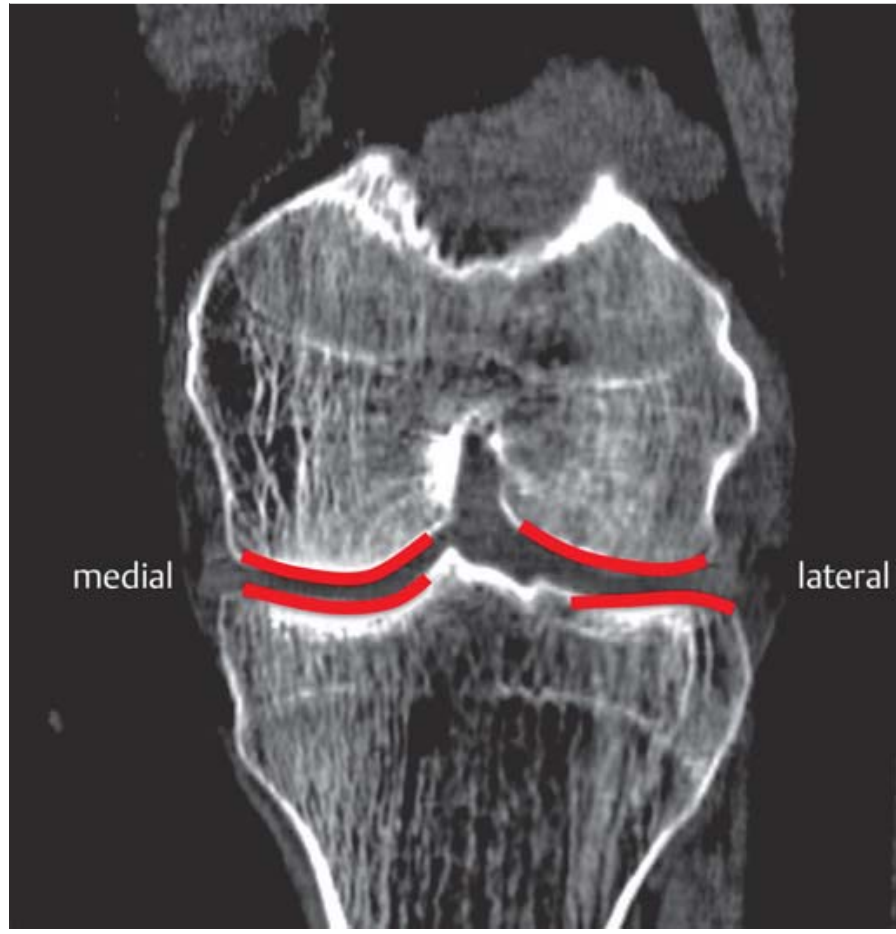

Abb. 2 Konvexität und Konkavität der Gelenkpartner.

Die das Kniegelenk umspannende Muskulatur stabilisiert und führt aktiv die Kinematik (Mm. quadriceps, biceps femoris, popliteus, gastrocnemii, semitendinosus, semimembranosus, gracilis). In der Folge kommt es zu einer der Beweglichkeit der Menisci angepassten Kinematik mit medial geringerer Translation als lateral (sogenanntes mediales Pivotieren) [2,3]. Dies entspricht einer zunehmenden Innenrotation der Tibia bei Flexion des Kniegelenks.

Vice versa kommt es strecknah zu einer Außenrotation der Tibia gegenüber dem Femur (sogenannte Schlussrotation). Diese verriegelt das Kniegelenk und ermöglicht den kraftsparenden Stand $[4,5]$.

Aktuelle Arbeiten zeigen, dass die beschriebene Kinematik einen Durchschnitt darstellt, es jedoch erhebliche interindividuelle Variabilität bis hin zur Umkehr mit lateralem Pivotieren gibt [6].

\section{Prinzipien}

\section{Femorotibialer Anpressdruck}

Während des normalen Ganges entspricht die femorotibiale Kontaktkraft dem 2- bis 3-fachen Körpergewicht [7-9]. Der resultierende Anpressdruck verteilt sich dabei physiologischerweise asymmetrisch zwischen dem medialen und lateralen Gelenkkompartiment [7]: $>>70 \%$ medial,

$><30 \%$ lateral.

Die Ursache liegt in einem Adduktionsmoment durch den wandernden, aber während des Gangzyklus stets medial der Tragachse (Miculizc-Linie) liegenden Körperschwerpunkt ( Abb. 3) [10-12]. Die Tatsache, dass das mediale Gelenkkompartiment bei gerader Beinachse stets mehr belastet wird als das laterale, wird als Ursache der medial wesentlich häufiger als lateral betonten Gonarthrose diskutiert [13-16].

\section{Epidemiologie}

\section{$\nabla$}

Aufgrund einer Arthrose des Kniegelenks wurden 2011 nach der Krankheitsartenstatistik der gesetzlichen Krankenversicherung 115000 Patienten krankgeschrieben. Dies entsprach knapp 5 Millionen Krankheitstagen [17].

Die Lebenszeitprävalenz der Gonarthrose beträgt bei über 55-jährigen Personen $18 \%$ und bei über 65-jährigen 23\% [1]. Hauptrisikofaktor der Gonarthrose ist dabei neben dem Alter die Adipositas $[13,14,18-20]$. Eine varische Beinachse führt zu einer beschleunigten Progression einer
Es gibt in der Kinematik erhebliche interindividuelle Variabilität bis hin zur Umkehr mit lateralem Pivotieren.
- Hauptrisikofaktoren der Gonarthrose sind Alter und Adipositas. 


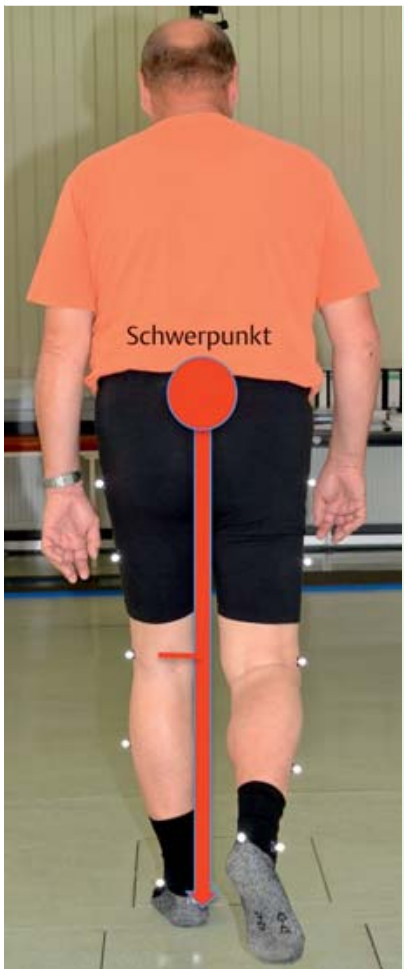

Abb. 3 Das Adduktionsmoment resultiert aus der relativ zur Beinachse medialen Position des Körperschwerpunkts während des Gangzyklus.

bereits vorhandenen medialen Arthrose [21-23]. Es ist jedoch fraglich, ob eine varische Beinachse zu einer Initiierung einer medialen Gonarthrose führt und damit eine Präarthrose darstellt $[21,24]$. Dagegen sprechen Hinweise, dass es ein konstitutionelles Varus gibt, welches kein unabhängiger Risikofaktor für die Entwicklung einer medialen Gonarthrose ist [25].

In Deutschland ist die operative Therapie der Wahl laut einer Umfrage [26]

> in $81 \%$ der Fälle eine Totalendoprothese,

- in $12 \%$ der Fälle eine Umstellungsosteotomie (HTO) und

- in 7\% der Fälle ein unikondylärer Gelenkersatz.

Im Gegensatz dazu spielt in den USA die HTO mit einem Verhältnis von $1: 17$ verglichen mit dem unikondylären Ersatz eine untergeordnete Rolle [27].

\section{Diagnostisches Vorgehen}

\section{$\nabla$}

\section{Anamnese}

Die Diagnostik der medialen Gonarthrose beginnt stets mit der Anamnese, wobei hier insbesondere eine genaue Schmerzanamnese zielführend ist (s. Übersicht).

\section{Zielgerichtete Diagnostik}

\section{Anamnese bei Verdacht auf Gonarthrose}

- Beschwerdedauer

- Belastungsabhängigkeit

- typischer Anlaufschmerz

- Nacht-/Ruheschmerz

- Ansprechen auf Analgetika und/oder intraartikuläre Injektionen

- stadienhafter Verlauf mit arthritischen Schüben und Ruhephasen

\section{Klinische Untersuchung}

Es folgt die klinische Untersuchung mit Ermittlung von

- Bewegungsausmaß,

- mediolateraler und anteroposteriorer Stabilität und

- Identifikation druckschmerzhafter Bereiche. 
Hierbei ist es entscheidend, intra- von extraartikulären Schmerzursachen zu differenzieren, die im Rahmen einer initialen Arthrose häufig begleitend auftreten; u. a.:

- Ansatztendinopathie von Pes anserinus,

- Tuberculum Gerdyi,

- M. biceps femoris,

M. adductor magnus

Die orientierende Untersuchung von Hüftgelenk und Wirbelsäule ist notwendig, um in das Kniegelenk ausstrahlende Schmerzen auszuschließen.

\section{Zielgerichtete Diagnostik}

\section{Tipp}

Bei unklaren Befunden kann im Einzelfall eine diagnostische Infiltration oder intraartikuläre Injektion in der Differenzialdiagnostik hilfreich sein.

\section{Radiologische Untersuchung \\ Röntgen}

Die radiologische Diagnostik beinhaltet eine Aufnahme a.-p. und seitlich im Stand. Idealerweise sollte eine Ganzbeinaufnahme erfolgen, um

- Achsabweichungen festzustellen,

- extra- von intraartikulären Fehlstellungen zu differenzieren und auch

- eine Operationsplanung ohne erneute Strahlenbelastung durchführen zu können.

Gerade bei initialer Gonarthrose ermöglicht die Rosenberg-Aufnahme ( $\nabla$ Abb.4) eine höhere diagnostische Sensitivität [28]. Bei der klassischen Standaufnahme befindet sich der Körperschwerpunkt über dem Kniegelenk, sodass ein geringer femorotibialer Kontaktdruck den Gelenkspalt definiert. Im Gegensatz dazu wird der Körperschwerpunkt bei der Rosenberg-Aufnahme weit nach posterior verlagert, sodass eine deutlich höhere Gelenkkontaktkraft resultiert. Auf den Nativbildern kann der Arthrosegrad nach Kellgren (Grad I-IV) klassifiziert werden [29].

Gehaltene Aufnahmen können zur Differenzierung zwischen Defekt- und echter ligamentärer Instabilität hilfreich sein. Dies unterstützt die Differenzialindikation zwischen unikondylärer und Totalendoprothese sowie die Bestimmung des mindestens erforderlichen Kopplungsgrads. Patella-axial- oder -défilé-Aufnahmen ermöglichen die Evaluation des patellofemoralen Gelenks. Dabei werden Arthroseausmaß, Patella Tilt und Shift bestimmt. Die Indikation zur Durchführung dieser Aufnahmen besteht bei

- patellofemoraler Symptomatik,

- klinischem V.a. Lateralisation der Patella,

Z Z.n. patellazentrierenden Voroperationen.

Die streng seitliche Aufnahme in $90^{\circ}$-Flexion erlaubt über das Arthrosemuster eine indirekte Abschätzung der Kniegelenkskinematik.

- Die bei erhaltener Kinematik (mediales Pivoting) typische Arthrose beginnt im mittleren bis vorderen Anteil (sogenannte anteromediale Arthrose, O Abb. 6). Bei Sklerose des mittleren und vorderen medialen Tibiaplateaus bleibt der Gelenkspalt zwischen posteromedialem Femur und medialer Tibiahinterkante erhalten [58,59].

- Im Gegensatz dazu entgleist die Kniegelenkskinematik bei Insuffizienz des VKB und führt zu einer posteromedialen Arthrose ( Abb. 7) $[58,59]$.

\section{Magnetresonanztomografie}

Ein MRT ist nur in Ausnahmefällen sinnvoll, da es über das Nativbild und die klinische Untersuchung kaum Zusatzinformationen liefert, welche die Therapieentscheidung beeinflussen. Stehen im Vordergrund der Beschwerdesymptomatik jedoch nicht Arthrosebeschwerden, ist ein MRT differenzialdiagnostisch wertvoll bei der Detektion von

- Meniskusläsionen,

- Knochenmarködem,

- Osteonekrose,

- Kreuzbandruptur,

- Synovialitis etc.
- Bei der klinischen Diagnostik müssen intraartikuläre von extraartikulären Schmerzursachen unterschieden werden.

- Radiologische Diagnostik bei V.a. Gonarthrose: Aufnahme a.-p. und seitlich im Stand, möglichst Ganzbeinröntgenaufnahme, möglichst Rosenberg-Aufnahme. 

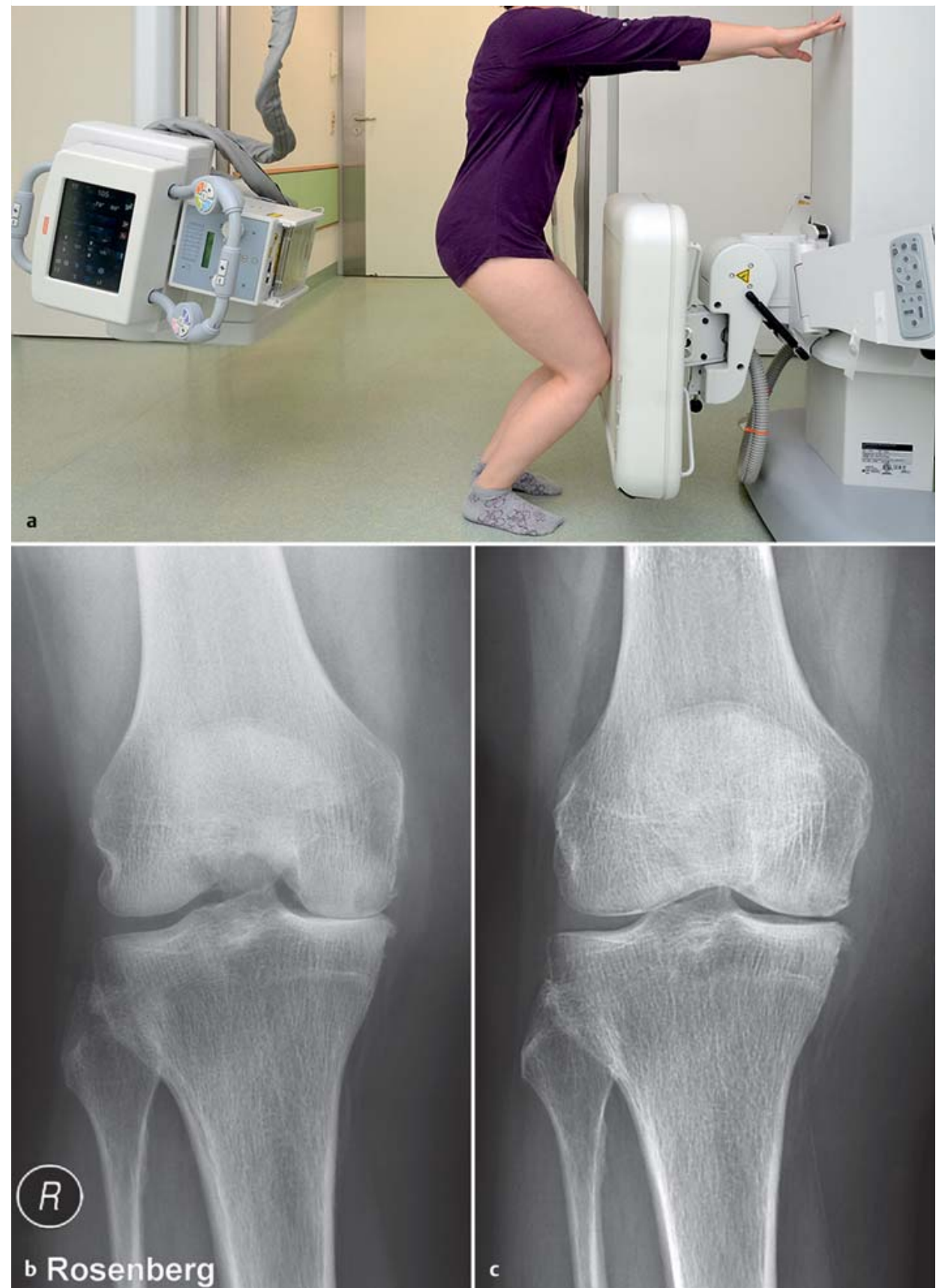

Abb. 4a bis c Rosenberg-Aufnahme. a Technik der Rosenberg-Aufnahme. b Bild einer medialen Gonarthrose in der Rosenberg-Aufnahme. c Anhand der a.-p. Standaufnahme desselben Kniegelenks würde der Arthrosegrad unterschätzt werden.

\section{Therapeutisches Vorgehen}

\section{$\nabla$}

Die Therapie der medialen Gonarthrose erfolgt stadiengerecht und beginnt in der Regel mit der konservativen Therapie.

\section{Konservative Therapie}

Die konservative Therapie der Gonarthrose entsprechend den aktuellen Empfehlungen der OARSI [101] ist in der Übersicht dargestellt.

\section{Schuhaußenranderhöhung}

Eine laterale Schuhaußenranderhöhung führt über eine dynamische Valgisierung während des Ganges zu einer signifikanten, aber geringen Reduktion des Adduktionsmoments im Gegensatz zu valgisierenden Orthesen, die keinen Effekt auf das Adduktionsmoment haben [30]. Aufgrund 


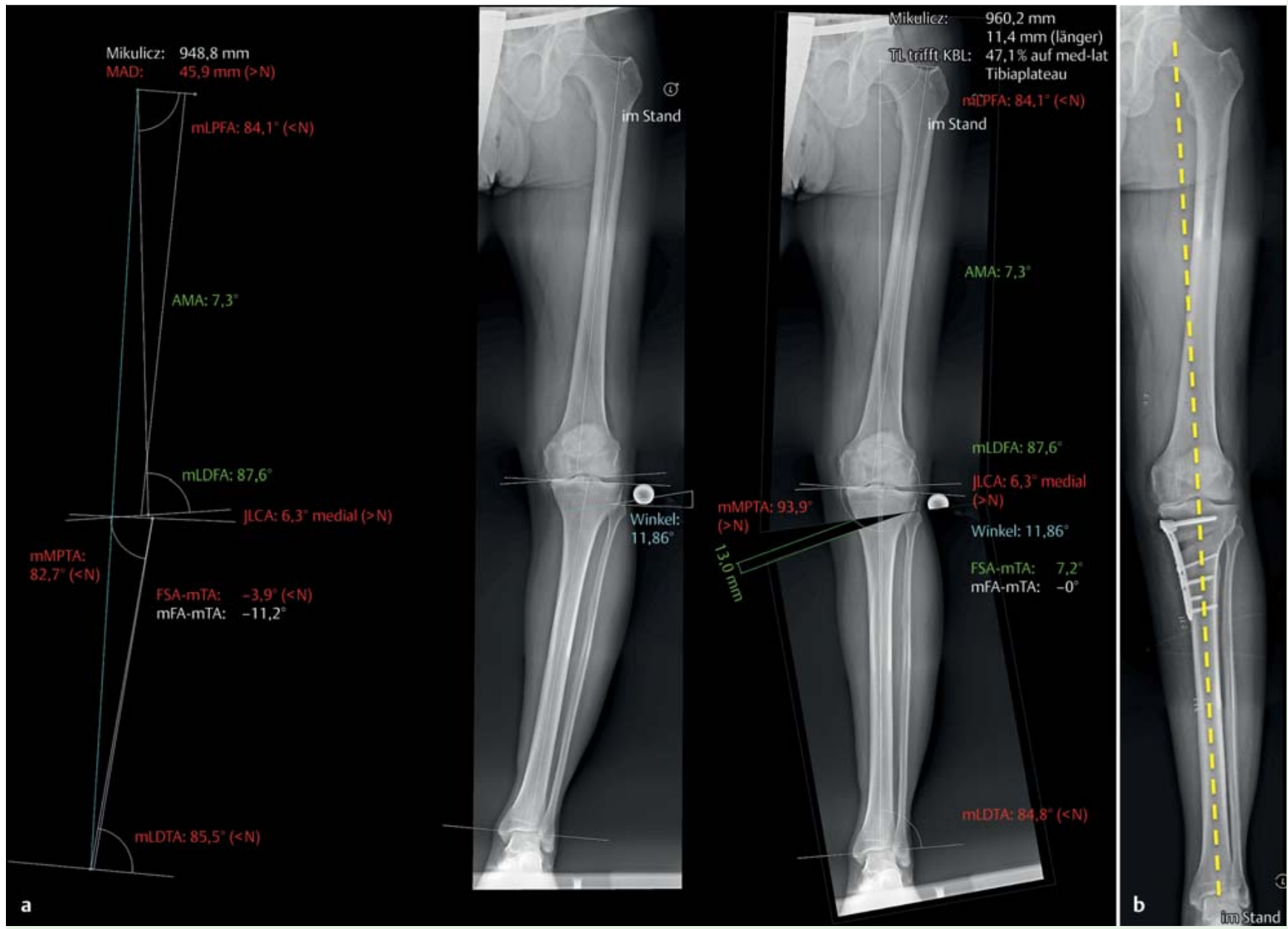

Abb. 5 a und $\mathbf{b}$ Varische Beinachse mit CORA (Center of Rotation and Angulation) im Bereich des Tibiakopfs. a Planung der Umstellungsosteotomie. AMA = Winkel zwischen anatomischer und mechanischer Achse, FSA-mTA = Winkel zwischen der anatomischen Femurachse und der mechanischen Tibiaachse, JLCA = Gelenklinienkonvergenzwinkel, KBL = Kniebasislinie, LDFA = lateraler distaler Femurwinkel, MAD = mechanische Achsabweichung, mFA-mTA = Winkel zwischen der mechanischen Femurachse und der mechanischen Tibiaachse, $\mathrm{mLDFA}=$ mechanischer lateraler distaler Femurwinkel, mLDTA = lateraler distaler Tibiawinkel, $\mathrm{mLPFA}=$ mechanischer lateraler proximaler Femurwinkel, $\mathrm{mMPTA}=$ mechanischer medialer proximaler Tibiawinkel (Normwerte jeweils $\left.87^{\circ} \pm 3^{\circ}\right), \mathrm{N}=\mathrm{Norm}-$ werte, $\mathrm{TL}=$ Traglinie oder Mikulicz-Linie. $\mathbf{b}$ Korrektur der Beinachse nach Umstellungsosteotomie (HTO).

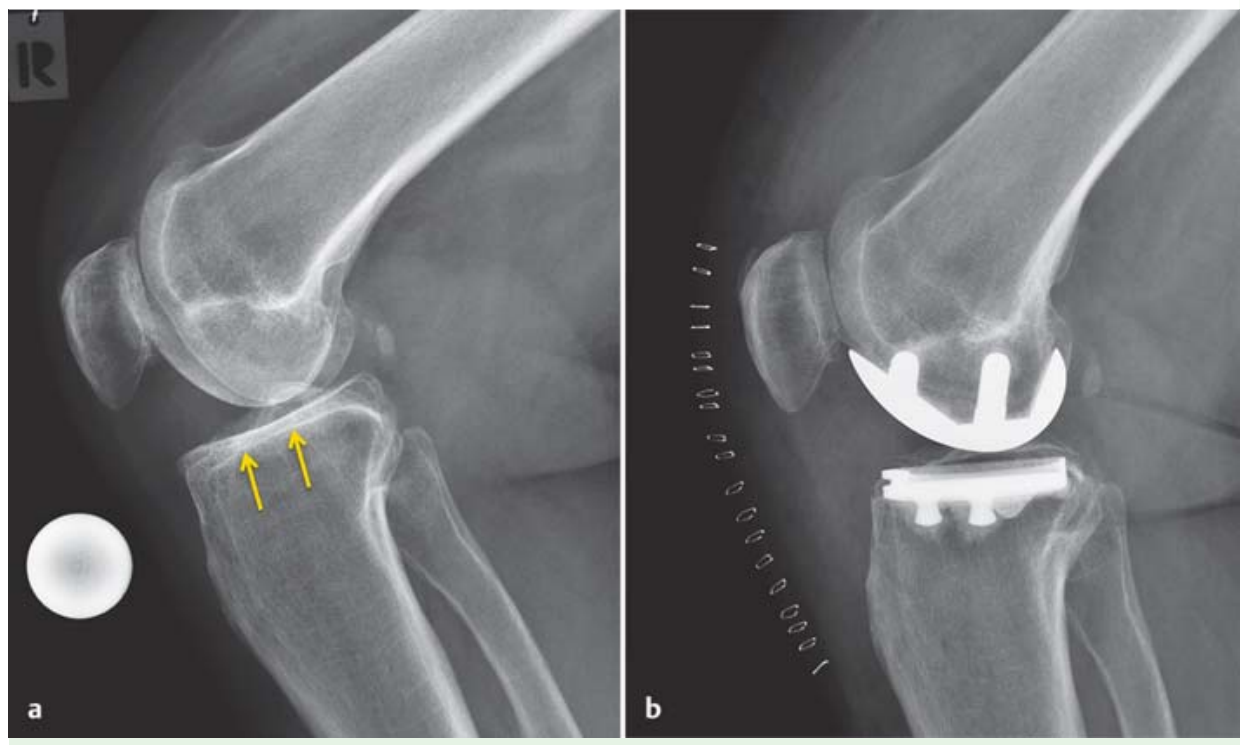

Abb. 6 a und $\mathbf{b}$ Anteromediale Arthrose als Zeichen eines intakten vorderen Kreuzbands. a Präoperatives Röntgenbild (Pfeile: arthrotische Veränderungen). b Unikondylärer Gelenkersatz mit fixer Plattform. 


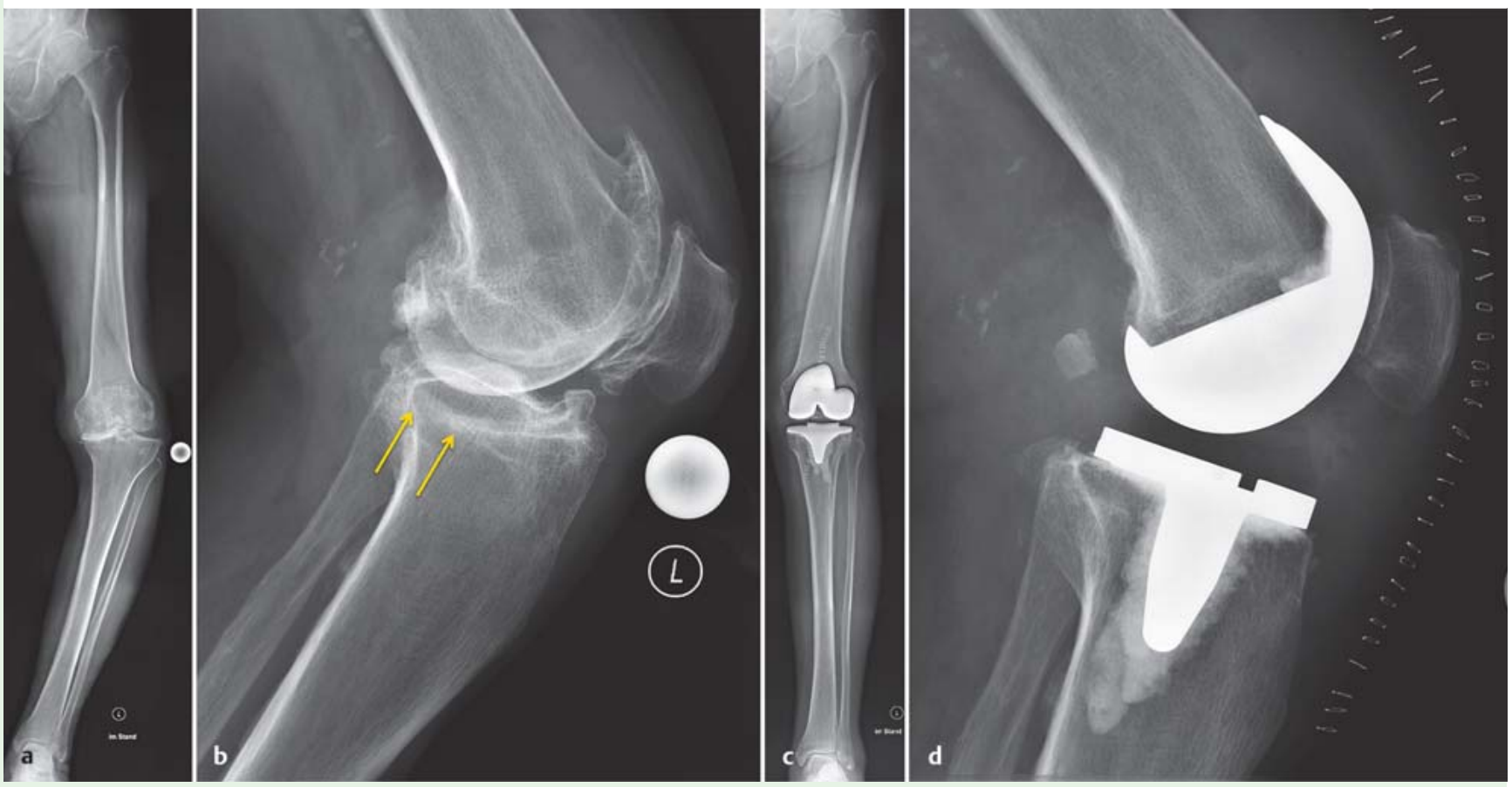

Abb. 7 a bis d Posteromediale Arthrose als Folge einer entgleisten Kniegelenkskinematik. a Präoperatives Röntgenbild (a.-p. Strahlengang). b Präoperatives Röntgenbild seitlich: Die Pfeile zeigen die posteriore Sklerose bzw. den posterioren Defekt. c, d Totalendoprothetischer Gelenkersatz.

des geringen klinischen Einflusses wird in einer aktuellen Cochrane-Analyse keine Empfehlung für diese Verfahren gegeben [31].

Übersicht
Konservative (Therapie-)Maßnahmen bei Gonarthrose
- Physiotherapie
- Kewichtsreduktion
- Schuhaußentenkorthese
- systemische medikamentöse Therapie
- intraartikuläre medikamentöse Therapie
(entsprechend den aktuellen Empfehlungen der OARSI)

Medikamentöse Therapie

Die intraartikuläre medikamentöse Therapie (Kortikosteroide und Hyaluronsäure) ist in einer aktuellen Metaanalyse der systemischen Therapie mit nichtsteroidalen Antiphlogistika (NSAID) überlegen, wobei offen bleibt, ob dies Folge eines größeren Placeboeffekts durch die Invasivität der Therapie ist [32-34].

\section{Cave}

\section{Kortikosteroidinjektion}

Problematisch erscheint dabei jedoch die Tatsache, dass eine vorhergehende intraartikuläre Therapie mit Kortikosteroiden das Infektionsrisiko bei folgender Endoprothesenimplantation deutlich erhöht [35].

\section{Arthroskopie}

Obwohl in der Literatur sehr umstritten, werden arthroskopische Verfahren auch ohne Begleitpathologie oft bei medialer Gonarthrose durchgeführt [36-38]. Débridement und Lavage führen zu einer allenfalls kurzfristigen Symptombesserung $[39,40]$. 
Tab. 1 Differenzialindikation zwischen tibialer Umstellungsosteotomie, unikondylärer und Totalendoprothese (in Anlehnung an [102]).

\begin{tabular}{|llll} 
& HTO & UNI & TEP \\
\hline Arthrose ${ }^{\circ}$ IV nach Kellgren & - & + & + \\
\hline Seitenbandinstabilität & $0 /+$ & - & + \\
\hline insuffizientes VKB oder HKB & + & + & + \\
\hline Defektinstabilität & - & - & + \\
\hline varische Beinachse $\left(>5^{\circ}\right)$ & + & - & + \\
\hline valgische Beinachse $\left(>5^{\circ}\right)$ & - & + & + \\
\hline gerade Beinachse $\left( \pm 5^{\circ}\right)$ & 0 & - & + \\
\hline symptomatische patellofemorale Arthrose & $0 /-$ & - & + \\
\hline Streckdefizit $\left(>10^{\circ}\right)$ & $0 /-$ & - & + \\
\hline Beugedefizit $\left(<90^{\circ}\right)$ & 0 & + & + \\
\hline biologisches Alter $>60$ Jahre & - & + & $0 /-$ \\
\hline Risikofaktoren der Knochenheilung & - & 0 & + \\
\hline hoher Patientenanspruch an Funktion/Sport & + & & + \\
\hline
\end{tabular}

+ gute Indikation; 0 weniger gute Indikation; - schlechte Indikation

$\mathrm{HKB}=$ hinteres Kreuzband, $\mathrm{HTO}=$ Umstellungsosteotomie, TEP = Totalendoprothese, UNI = unikondyläre Prothese,

$\mathrm{VKB}=$ vorderes Kreuzband

\section{Knorpelchirurgie}

Ebenfalls kurzfristige Erfolge sind nach knorpelchirurgischen Eingriffen bei medialer Arthrose zu erwarten [41-43]. Dies steht im Gegensatz zu den guten Ergebnissen der Knorpelchirurgie bei umschriebenen Knorpeldefekten (Osteochondrosis dissecans, traumatisches Knorpel-Flake) ohne relevante Arthrose, die sich durch einseitige Lokalisation (nur femoral oder tibial) auszeichnen [44-46].

\section{Operative Therapie}

Im Folgenden sollen nur die im Langzeitergebnis der Arthroskopie überlegenen Operationsverfahren wie Umstellungsosteotomie, unikondyläre und bikondyläre Endoprothese detaillierter dargestellt werden.

Die Differenzialindikation zwischen diesen Verfahren ist nicht immer klar zu definieren. Zur Orientierung werden gute, weniger gute und schlechte Indikationen der Verfahren in \ Tab. 1 wiedergegeben.

Bei klinisch symptomatischer patellofemoraler Arthrose wird die Implantation einer UNI von den meisten Autoren kritisch gesehen. Die Kombination mit einem zusätzlichen patellofemoralen Ersatz oder alternativ bikompartimentelle Prothesen, die das mediale und patellofemorale Gelenk unter Erhalt des lateralen Kompartiments ersetzen, stellen hier eine Option zur Vermeidung einer TEP dar. Die Datenlage zu diesen Implantaten ist jedoch aufgrund des geringen Einsatzes äußerst beschränkt.

Auch nach kritischer Wertung der in @ Tab. 1 genannten Kriterien verbleibt in vielen Fällen eine Schnittmenge der Operationsverfahren, die eine individuelle Abwägung gemeinsam mit dem Patienten unter Berücksichtigung weicher, wissenschaftlich nicht unterlegter Faktoren (Erwartungshaltung, berufliches Umfeld, Kosmetik, Aktivitätsniveau) erfordert [47].

Wenn ein extraartikulärer Achsfehler vorliegt, liegt das Maximum des Fehlers („center of rotation and angulation", CORA) in der Regel im Bereich der proximalen Tibia, sodass die hohe tibiale Osteotomie (HTO) zum Standardverfahren in der gelenkerhaltenden Chirurgie des Kniegelenks geworden ist ( $\bullet$ Abb. 5) $[48,49]$. Dennoch kann in seltenen Fällen in Abhängigkeit des CORA und der Gelenkebene auch eine femorale oder aber eine Doppelosteotomie von Femur und Tibia indiziert sein [50].

Ein moderates Streckdefizit kann bei der HTO durch Reduktion des tibialen Slope (nicht jedoch bis zum anterioren Slope) therapiert werden. Eine posteriore Instabilität durch Insuffizienz des hinteren Kreuzbands kann durch Erhöhung des tibialen Slope gemildert werden, wobei $10^{\circ}$ nicht wesentlich überschritten werden sollten [51]. Im Gegensatz dazu führt eine Verringerung des tibialen Slope bei Insuffizienz des vorderen Kreuzbands nicht zu einer Reduktion der vorderen Schublade, jedoch einer geringen Zunahme der rotatorischen Stabilität (Pivot-Shift-Test) [52].

Die Differenzialindikation zwischen den Verfahren tibiale Umstellungsosteotomie (HTO), unikondyläre (INI) und Totalendoprothese (TEP) ist nicht immer klar zu definieren. 
- Die Datenlage hinsichtlich des Outcomes nach Verwendung einer Blutsperre und einer Wunddrainage zeigt zu geringe Unterschiede, als dass diese beiden Maßnahmen generell empfohlen werden könnten.

- Die perioperative Gabe von Tranexamsäure reduziert den Blutverlust und die Erfordernis von Transfusionen.
- Therapeutische Ziele bei der TEP-Operation: zur mechanischen Achse von Femur und Tibia jeweils senkrechte Resektion, Release der Weichteile $(\rightarrow$ Erzielung eines symmetrischen Streckspalts).
- Therapeutische Möglichkeiten der unikondyläre Endoprothese (UNI): mobile Plattform $\rightarrow$ ein in Beugung und Streckung stabiles mediales Kompartiment; der distale femorale Gelenkwinkel wird jedoch nicht rekonstruiert, fixe Plattform $\rightarrow$ anatomische Resektionen mit Instabilität der Spalten (belassen oder durch das Inlay ausgleichen); der proximale tibiale Gelenkwinkel ist variabel.
Die bisherige Evidenz zum teilendoprothetischen Ersatz bei Insuffizienz des vorderen Kreuzbands ist limitiert, sodass dieses Verfahren bislang nicht als Standard empfohlen werden kann $[53,54]$. Die überwiegende Mehrzahl der Autoren sieht darin aufgrund der resultierenden pathologischen Kniegelenkskinematik eine relative Kontraindikation für einen Teilersatz [55-57].

\section{Operationsverfahren}

Die Verwendung einer Blutsperre ist bei inkonsistenter Evidenz aufgrund der besseren Übersicht in vielen Kliniken Standard. Die detektierbaren Unterschiede hinsichtlich Blutverlust, postoperativem Schmerz, Komplikationen, Funktion und Zementierqualität sind gering [60,61]. Ähnlich kontrovers ist die Datenlage zu Wunddrainagen nach unikondylärer oder Totalendoprothese. In der Literatur dargestellte Unterschiede sind gering, sodass keine generelle Empfehlung für die routinemäßige Verwendung von Drainagen gegeben werden kann [62-64].

Im Gegensatz dazu konnte in zahlreichen Arbeiten konsistent gezeigt werden, dass die perioperative Gabe von Tranexamsäure den Blutverlust und die Erfordernis von Transfusionen reduziert, ohne dabei die Komplikationsrate zu erhöhen [65]. Wenngleich die Datenbasis dafür geringer ist, führt auch die intraartikuläre Applikation von Tranexamsäure zu einer Reduktion des Blutverlusts bei kniechirurgischen Eingriffen [66].

\section{OP-Technik Totalendoprothese (TEP)}

Der operative Standardzugang ist medial parapatellar. Er ermöglicht die beste Exposition des Gelenks und ist problemlos erweiterbar. Im Rahmen minimalinvasiver Operationstechniken haben sich der mediale Subvastus- und Midvastus-Zugang etabliert. Sie zeigen in Metaanalysen bis zu 1 Jahr nach Operation zwar geringe funktionelle Vorteile gegenüber dem Standardzugang, erfordern aber eine eigene Lernkurve [67-69].

Den gängigen Operationsphilosophien gemein sind die zur mechanischen Achse von Femur und Tibia jeweils senkrechte Resektion und das Release der Weichteile zur Erzielung eines symmetrischen Streckspalts. Der anzustrebende posteriore Slope der Tibia sollte den Empfehlungen des Herstellers folgen und variiert daher in Abhängigkeit des verwendeten Implantats und des Erhalts oder der Resektion des hinteren Kreuzbands zwischen $0^{\circ}$ und $7^{\circ}$.

In der Festlegung des Beugespalts scheiden sich die Operationsphilosophien:

- Bei der gemessenen Resektionstechnik („Femur first“) wird die Rotation des Femurteils anhand knöcherner Landmarken (Epikondylen, Whiteside Line, posteriore Kondylen) ausgerichtet. Die Symmetrie und Weite des Beugespalts werden durch anschließendes Weichteil-Release produziert.

- Im Gegensatz dazu wird bei der weichteilorientierten Technik („Tibia first“) die Rotation des Femurteils so eingestellt, dass ein symmetrischer Beugespalt resultiert, sodass weitgehend auf ein Release des Beugespaltes verzichtet werden kann. Die Weite dieses Beugespaltes wird dann über die Variabilität der distalen Femurresektion auf den Streckspalt übertragen.

\section{OP-Technik unikondyläre Endoprothese (UNI)}

Die Zugänge entsprechen denen der TEP (s. o.), wobei sich beim UNI aufgrund der kleineren erforderlichen Exposition die minimalinvasiven Zugänge durchgesetzt haben. Im Gegensatz zur TEP erfolgt prinzipiell kein Weichteil-Release. Lediglich durch die Entfernung von Osteophyten wird die Bandspannung indirekt beeinflusst.

Wie bei der TEP werden 2 unterschiedliche Operationsphilosophien unterschieden.

- Bei Verwendung einer mobilen Plattform wird der bestmöglichen Stabilität der Vorzug über die perfekte Rekonstruktion der femoralen und tibialen Gelenkebene gegeben. Nach der mechanischen senkrechten anatomischen Resektion des medialen Tibiaplateaus wird der Beugespalt durch eine ebenfalls anatomische Resektion des posterioren Femurs definiert. Die Höhe dieses Spaltes wird dann gemessen und auf die Streckung im Sinne einer variablen distalen Femurresektion übertragen. Dadurch resultiert regelhaft ein in Beugung und Streckung stabiles mediales Kompartiment, der distale femorale Gelenkwinkel wird jedoch nicht rekonstruiert.

- Im Gegensatz dazu wird bei fixen Plattformen meist eine gemessene Resektionsstrategie verfolgt. Hier erfolgen sowohl tibial als auch femoral distal und posterior anatomische Resektionen. Eine resultierende Instabilität der Spalten wird in Grenzen hingenommen oder durch das Inlay ausgeglichen, sodass bei dieser Technik der proximale tibiale Gelenkwinkel variabel ist. Beiden Techniken gemein ist das Ziel, den tibialen Slope bestmöglich zu rekonstruieren. 


\section{OP-Schritte und Tricks}

Tipp

Die bestmögliche Kontrolle ist die Evaluation des tibialen Resektats, das daher möglichst in toto geborgen werden sollte.

\section{OP-Technik tibiale Korrekturosteotomie (HTO)}

Im Folgenden soll die weit überwiegend verbreitete Technik der medial öffnenden biplanaren Osteotomie beschrieben werden.

Der operative Zugang besteht aus einer schrägen Hautinzision oberhalb des Pes anserinus. Er ermöglicht ein Arbeiten der oszillierenden Säge ohne kontaminierenden Hautkontakt und erlaubt das Einschieben einer winkelstabilen Platte.

Das oberflächliche Innenband wird regelhaft großflächig von der Tibia gelöst, um eine relative Verkürzung mit Hyperpression des medialen Gelenkkompartiments zu vermeiden. Der Pes anserinus sollte geschont werden, da einerseits lokale Beschwerden resultieren können und er andererseits den Ansatzpunkt für aktive mediale und rotatorische Stabilisatoren des Kniegelenks darstellt.

Nach Umfahren der Tibia mit Hohmann-Haken erfolgt eine biplanare Resektion unter Aussparung der Tuberositas tibiae. Liegt präoperativ bereits eine Patella baja vor, empfiehlt sich eine nach distal auslaufende Resektion der Tuberositas, um einen weiteren Tiefstand der Patella zu verhindern. Ansonsten wird die aufsteigende Resektion der Tuberositas empfohlen. Die quere Resektion der Tibia sollte bis knapp vor die laterale Kortikalis erfolgen, ohne diese jedoch zu durchtrennen. In der Folge wird der Osteotomiespalt langsam aufgedehnt und nach geplanter Korrektur winkelstabil fixiert. Um die besten Ergebnisse zu erzielen, wird eine moderate Überkorrektur der Beinachse auf $2^{\circ}$ bis maximal $5^{\circ}$ Valgus empfohlen [70].

Da die Variation des posterioren Slope der Tibia bei der HTO in Grenzen die Adressierung unterschiedlicher Pathologien ermöglicht (vordere oder hintere Instabilität, Streckdefizit), muss der Sagittalebene die gleiche intraoperative Aufmerksamkeit wie der Frontalebene zuteil werden.

\section{Nachbehandlung}

\section{Schmerzhemmung}

Es existieren unterschiedliche analgetische Nachbehandlungskonzepte. In den letzten Jahren populär geworden ist die durch den Operateur intraoperativ durchzuführende lokale Infiltrationsanästhesie (LIA) [71].

Postoperativ werden verschiedene Systeme eingesetzt:

- Systeme, die den Schmerz im Bereich der Entstehung hemmen

$\checkmark$ intraartikuläre Analgetikapumpen

- Systeme, welche die Nervenfortleitung hemmen

$\checkmark$ N.-femoralis-Katheter und/oder

- N.-ischiadicus-Katheter

- zentral schmerzhemmede Systeme

> patientenkontrollierte Opioidapplikation (PCA)

Periphere Nervenkatheter sind dabei bezüglich der Schmerzreduktion der systemischen Analgesie überlegen [72]. LIA wiederum ist in einer aktuellen Metaanalyse peripheren Katheterverfahren überlegen und hat den Vorteil, dass keine motorischen Defizite die Mobilisation behindern [71].

\section{Thromboseprophylaxe}

Die AWMF empfiehlt entsprechend ihrer aktuellen S3-Leitlinie eine medikamentöse Thromboseprophylaxe nach kniegelenksnahen Eingriffen von 11-14 Tagen [73].

\section{Physikalische Therapie und Bewegungstherapie}

Die in der klinischen Praxis übliche postoperative Kryotherapie führt in einer Metaanalyse zu einer Reduktion von Blutverlust, Schmerz sowie einer Verbesserung der Funktion, wobei diese Effekte gering und inkonsistent sind [74].

CPM-Schienen werden regelhaft zur Verbesserung der postoperativen Beweglichkeit eingesetzt, wobei eine Evidenz für diese Verfahren fehlt [75].

- In der Schmerzhemmung ist LIA (lokale Infiltrationsanästhesie) der systemisch und peripher wirksamen Schmerzhemmung überlegen. 
- Mögliche Komplikationen: Fraktur der lateralen Kortikalis, Pseudarthrose, Korrekturverlust/ Implantatlockerung, Infektion.

- Der unikondyläre Gelenkersatz (UNI) ist das mit Abstand komplikationsärmste Verfahren verglichen mit HTO und TEP, die eine vergleichbare Komplikationsrate aufweisen.

- Die TEP zeigt signifikant bessere Langzeitergebnisse als UNI und HTO.

\section{Belastungslimitierung}

Nach Teil- oder Totalendoprothese des Kniegelenks ist eine unmittelbare Vollbelastung an Unterarmgehstützen möglich. Bei der HTO führt die frühe Vollbelastung 2 Wochen nach Operation zu funktionell besseren Ergebnissen als die historisch empfohlene Teilbelastung von 6 Wochen [76]. Kommt es jedoch zu einer Fraktur der lateralen Tibiakortikalis, empfiehlt sich eine verzögerte Aufbelastung, um Korrekturverlust, Implantatversagen oder -lockerung zu vermeiden.

\section{Komplikationen}

Nach medial öffnender HTO beträgt das Risiko einer Fraktur der lateralen Kortikalis über 20\% [77].

Pseudarthrosen werden in Abhängigkeit der Füllung des Osteotomiespalts mit autologer Spongiosa (2,6\%), allogener Spongiosa (4,6\%) und synthetischem Knochenersatzmaterial $(4,5 \%)$ beobachtet [78]. Im Gegensatz dazu steht eine Pseudarthroserate von nur 1\% bei Verzicht auf eine Füllung des Osteotomiespalts und Verwendung dezidierter winkelstabiler Implantate [79].

Die Einführung solcher Implantate hat die ehemals typische Komplikation des Korrekturverlusts nach HTO bis in den Bereich der Messgenauigkeit der nativradiologischen Kontrolle gesenkt [79-81]. Nach UNI wird bei Verwendung von Implantaten mit mobiler Plattform in bis zu 3\% der Fälle eine Dislokation des Inlays beobachtet. In $1 \%$ der Fälle kommt es zu einer Frühlockerung der tibialen Komponente und in 0,3 - 2\% der Fälle zu einer Infektion [82-84].

Frakturen des medialen Tibiaplateaus sind hingegen sehr selten [85]. Die publizierte Infektionsrate nach TEP liegt ebenfalls zwischen 0,3 und $2 \%$ [86-89]. Das Risiko einer intraoperativen Fraktur nach TEP beträgt 0,2\% [88].

Eine Bewertung von HTO, UNI und TEP hinsichtlich ihrer Komplikationsrate ist aufgrund der abweichenden Methoden der unterschiedlichen Einzelstudien jedoch problematisch. In einer diese Verfahren vergleichenden Metaanalyse konnte gezeigt werden, dass das über alle Komplikationen gewichtet gemittelte relative Risiko von TEP:HTO:UNI 1,00:1,01:0,31 beträgt. Damit ist der unikondyläre Gelenkersatz das mit Abstand komplikationsärmste Verfahren verglichen mit HTO und TEP, die eine vergleichbare Komplikationsrate aufweisen [90].

\section{Ergebnisse}

Innerhalb der ersten 3 Monate nach Operation sind die funktionellen Ergebnisse nach UNI besser als nach HTO oder TEP, nach einem Jahr gleichen sich UNI und HTO an und bleiben der TEP überlegen [91-93]. Insbesondere die Rückkehr zu Freizeitaktivitäten und Sport ist nach TEP nicht so sicher möglich wie nach UNI oder HTO [94]. In einer intraindividuell vergleichenden Studie konnte eine Präferenz der Patienten für die Versorgung mittels UNI im Gegensatz zur TEP gezeigt werden [95].

Das Langzeitergebnis nach TEP ist dem UNI und der HTO signifikant überlegen. Es werden 10Jahres-Überlebensraten von über 95\% in großen Fallserien [96,97] und in Endoprothesenregistern angegeben [98]. In einer aktuellen Metaanalyse von vergleichenden Studien konnten keine Unterschiede im Langzeitergebnis nach HTO im Vergleich zum UNI mit dem Endpunkt Revision festgestellt werden, sodass die Verfahren diesbezüglich gleichwertig sind [47]. Die 10-JahresLiegeraten betrugen nach HTO 84,4\% und nach UNI 86,9\% [99].

Im Gegensatz dazu ist aber das Ergebnis nach Revision einer HTO auf eine TEP signifikant besser als nach UNI [100]. Beide Revisionsoptionen sind jedoch dem Ergebnis nach primärer TEP unterlegen [100].

\section{Interessenkonflikt}

$\nabla$

Die Autoren bestätigen, dass kein Interessenkonflikt vorliegt.

\section{Literatur}

Die Literatur ist in der Online-Version unter www.thieme-connect.de/ejournals einsehbar.

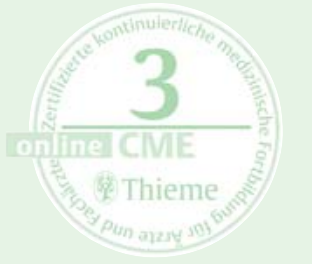




\section{CME-Fragen Die mediale Gonarthrose}

1 Wodurch ist die mediale Gonarthrose gekennzeichnet?

A Die mediale Gonarthrose ist häufiger als die laterale Gonarthrose.

B Die mediale Gonarthrose ist in der Regel Folge einer rheumatologischen Grunderkrankung.

C Die Lebenszeitprävalenz der medialen Gonarthrose von 65-Jährigen beträgt über $40 \%$.

D Männer sind deutlich häufiger von der medialen Gonarthrose betroffen als Frauen.

E Adipositas ist kein Risikofaktor für die Entwicklung einer Gonarthrose.

2 Welche Aussage zur Anatomie des Kniegelenks ist korrekt?

A Das mediale tibiale Gelenkkompartiment ist konvex.

B Der mediale Femurkondylus ist konkav.

C Das laterale tibiale Gelenkkompartiment ist konvex.

D Der laterale Femurkondylus ist konkav.

E Medialer und lateraler Femurkondylus sind regelhaft gleich groß.

3 Nur eine der folgenden Aussagen zur antero- bzw. posteromedialen Gonarthrose ist zutreffend. Welche?

A Eine anteromediale Gonarthrose spricht für ein intaktes vorderes Kreuzband.

B Eine anteromediale Gonarthrose spricht für ein insuffizientes Innenband.

C Eine anteromediale Gonarthrose spricht für eine posterolaterale Instabilität.

D Eine posteromediale Gonarthrose ist Zeichen einer intakten Gelenkskinematik.

E Alle medialen Gonarthrosen beginnen als anteromediale Arthrosen.

4 Wodurch sind die Korrekturmöglichkeiten der Instabilität im Kniegelenk gekennzeichnet?

A Eine anteriore Instabilität kann durch Erhöhung des posterioren tibialen Slope im Rahmen einer Korrekturosteotomie reduziert werden.

B Eine posteriore Instabilität ist eine absolute Kontraindikation für eine Korrekturosteotomie.

C Eine anteriore Instabilität ist eine absolute Kontraindikation für eine Korrekturosteotomie.

D Bei einer Korrekturosteotomie ist stets ein posteriorer Slope der Tibia von $0^{\circ}$ anzustreben.

E Eine posteriore Instabilität kann durch Erhöhung des posterioren tibialen Slope im Rahmen einer Korrekturosteotomie reduziert werden.
5 Welche Aussage zu den Operationsverfahren nach Umstellungsosteotomie (HTO) ist korrekt?

A Die klinischen Ergebnisse einer Knie-TEP nach HTO sind schlechter als nach unikondylärer Prothese (UNI).

B Die klinischen Ergebnisse einer Knie-TEP nach HTO sind besser als nach UNI.

c Die klinischen Ergebnisse einer Knie-TEP nach HTO sind gleich gut wie nach UNI.

D Die klinischen Ergebnisse einer Knie-TEP nach HTO sind genauso gut wie die einer primären Knie-TEP.

E Nach HTO ist immer die Implantation einer gekoppelten Endoprothese erforderlich.

6 Wodurch sind die Komplikationen nach Umstellungsosteotomie (HTO) gekennzeichnet?

A Korrekturverluste nach HTO werden mit modernen winkelstabilen Implantaten kaum noch beobachtet.

B Der Korrekturverlust ist die Hauptversagensursache der HTO.

C Zur Vermeidung eines Korrekturverlusts ist die Füllung des Osteotomiespalts mit Spongiosa erforderlich.

D Nach HTO ist eine Teilbelastung für mindestens 12 Wochen erforderlich.

E Die akzidentelle Fraktur der lateralen Kortikalis ist eine äußerst seltene Komplikation der HTO.

\section{Tranexamsäure...}

A ... beschleunigt die Durchbauung des Osteotomiespalts nach Umstellungsosteotomie.

B ... verhindert embolische Ereignisse bei der zementierten Implantation einer Totalendoprothese (TEP).

C ... ist, lokal appliziert, ein potentes Analgetikum.

D ... reduziert den perioperativen Blutverlust nach kniegelenksnahen Operationen.

E ...erhöht die Infektionsrate nach kniegelenksnahen Operationen.

\section{Wodurch ist die Rosenberg-Aufnahme gekennzeichnet?}

A Die Rosenberg-Aufnahme gibt Aufschluss über den Zustand des patellofemoralen Gelenks.

B Die Rosenberg-Aufnahme hat eine höhere Sensitivität als die Ganzbeinstandaufnahme in der Detektion der Gonarthrose.

C Die Rosenberg-Aufnahme wird im Liegen durchgeführt.

D Der Körperschwerpunkt befindet sich bei der RosenbergAufnahme vor der Körperlängsachse (Vertikalachse).

E Die Rosenberg-Aufnahme wird im Sitzen durchgeführt. 
Nur eine der folgenden Aussagen zum Adduktionsmoment des Kniegelenks ist zutreffend. Welche?

A 作 dass die Gelenkkontaktkraft im lateralen höher als im medialen Kompartiment ist.

B Das Adduktionsmoment des Kniegelenks lässt sich weder durch eine Kniegelenkorthese noch durch eine Schuhaußenranderhöhung verringern.

C Das Adduktionsmoment des Kniegelenks ist Ursache der gleichmäßigen Verteilung der Gelenkkontaktkraft zwischen medialem und lateralem Kompartiment.

D Das Adduktionsmoment des Kniegelenks wird durch die Beinachse und das Gangbild determiniert.

E Das Adduktionsmoment des Kniegelenks bleibt bei einer korrekt durchgeführten HTO konstant.
10

Welche Aussage zur Biomechanik ist korrekt?

A Der femorotibiale Anpressdruck entspricht beim normalen Gehen dem 1,5-fachen Körpergewicht.

B Die im Kniegelenk wirkende Gelenkkontaktkraft überschreitet nie die Bodenreaktionskraft.

C Der laterale femorotibiale Anpressdruck wird im Rahmen einer HTO durch eine Valgisation verringert.

D Extraartikuläre Korrekturosteotomien können prinzipiell die im Gelenk wirkenden Kräfte nicht verändern.

E Der femorotibiale Anpressdruck ist bei mechanisch gerader Beinachse im medialen deutlich höher als im lateralen Gelenkkompartiment.

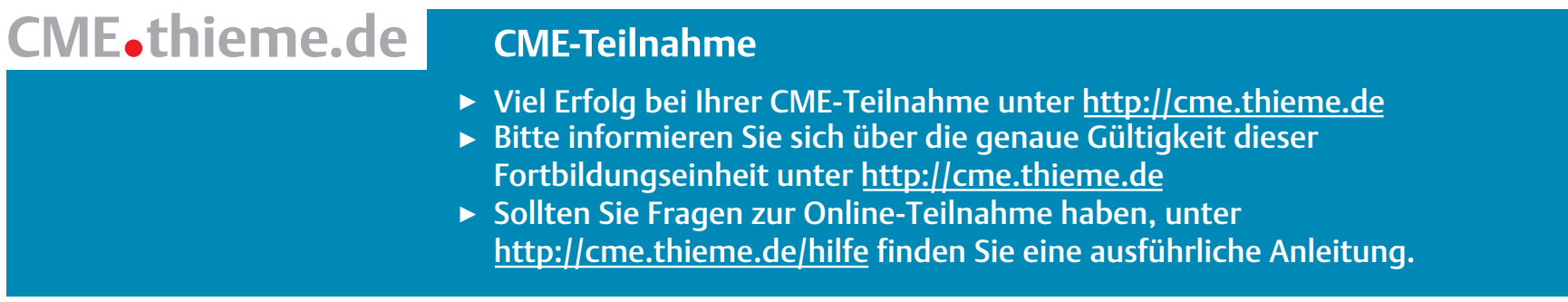

\section{Impressum}

$\nabla$

Refresher Orthopädie und Unfallchirurgie

Die mediale Gonarthrose

Autoren:

G. Matziolis, E. Röhner

Orthopädische Klinik, Friedrich-Schiller-Universität Jena, Campus Eisenberg

Konzeption und Umsetzung:

Joachim Ortleb

Georg Thieme Verlag KG

Klinik und Praxis

Zertifizierte Fortbildung
Für Angaben über Dosierungsanweisungen und Applikationsformen kann vom Verlag keine Gewähr übernommen werden. Jeder Benutzer ist angehalten, durch sorgfältige Prüfung der Beipackzettel der verwendeten Präparate und ggf. nach Konsultation eines Spezialisten festzustellen, ob die dort gegebene Empfehlung für Dosierungen oder die Beachtung von Kontraindikationen gegenüber der Angabe in dieser Broschüre abweicht. Eine solche Prüfung ist besonders wichtig bei selten verwendeten Präparaten oder solchen, die neu auf dem Markt gebracht worden sind. Jede Dosierung oder Applikation erfolgt auf eigene Gefahr des Benutzers.

\section{(c) 2015 Georg Thieme Verlag KG, Rüdigerstraße 14, 70469 Stuttgart}

Unsere Homepage: http://www.thieme.de

Printed in Belgium

Satz: Ziegler + Müller, text form files, Kirchentellinsfurt 